\title{
Nanonization of megestrol acetate by laser fragmentation in aqueous milieu
}

\author{
Jean-Philippe Sylvestre ${ }^{\mathrm{a}}$, Marie-Christine Tang ${ }^{\mathrm{b}}$, Alexandra Furtos ${ }^{\mathrm{b}}$, Grégoire Leclair $^{\mathrm{c}}$, \\ Michel Meunier ${ }^{\mathrm{a}}$ and Jean-Christophe Leroux ${ }^{\mathrm{c}, \mathrm{d}}$
}

\begin{abstract}
${ }^{\mathrm{a}}$ Canada Research Chair in Laser Micro/nano-engineering of materials, Laser Processing and Plasmonics Laboratories, Department of Engineering Physics, École Polytechnique de Montréal, P.O. Box 6079, Downtown Station, Montréal, Québec, H3C 3A7, Canada;

${ }^{\mathrm{b}}$ Regional Center for Mass Spectrometry, Department of Chemistry, Université de Montréal, P.O. Box 6128, Downtown Station, Montréal, Québec, H3C 3J7, Canada;

${ }^{c}$ Faculty of Pharmacy, Université de Montréal, P.O. Box 6128, Downtown Station, Montréal, Québec, H3C 3J7, Canada;

${ }^{\mathrm{d}}$ Institute of Pharmaceutical Sciences, Department of Chemistry and Applied Biosciences, ETH Zürich, Wolfgang-Pauli-Str. 10, HCI H 301, 8093, Zürich, Switzerland
\end{abstract}

Address all correspondence to:

Prof. Jean-Christophe Leroux, Institute of Pharmaceutical Sciences

ETH Zürich

Wolfgang-Pauli-Str. 10

HCI H 301

8093 Zürich

Switzerland

Tel: $+41(0) 446337310$

Fax: +41 (0) 446331311

Email address: jleroux@ethz.ch 


\begin{abstract}
Nanonization is a simple and effective method to improve dissolution rate and oral bioavailability of drugs with poor water solubility. There is growing interest to downscale the nanocrystal production to enable early preclinical evaluation of new drug candidates when compound availability is scarce. The purpose of the present study was to investigate laser fragmentation to form nanosuspensions in aqueous solution of the insoluble model drug megestrol acetate (MA) using very little quantities of the drug. Laser fragmentation was obtained by focusing a femtosecond ( $\mathrm{fs}$ ) or nanosecond (ns) laser radiation on a magnetically stirred MA suspension in water or aqueous solution of a stabilizing agent. The size distribution and physicochemical properties of the drug nanoparticles were characterized, and the in vitro dissolution and in vivo oral pharmacokinetics of a laser fragmented formulation were evaluated. A MA nanosuspension was also prepared by media milling for comparison purpose. For both laser radiations, smaller particles were obtained as the laser power was increased, but at a cost of higher degradation. Significant nanonization was achieved after a 30-min fs laser treatment at $250 \mathrm{~mW}$ and a 1-h ns laser treatment at $2500 \mathrm{~mW}$. The degradation induced by the laser process of the drug was primarily oxidative in nature. The crystal phase of the drug was maintained, although partial loss of crystallinity was observed. The in vitro dissolution rate and in vivo bioavailability of the laser fragmented formulation were similar to those obtained with the nanosuspension prepared by media milling, and significantly improved compared to the coarse drug powder. It follows that this laser nanonization method has potential to be used for the preclinical evaluation of new drug candidates.
\end{abstract}

Keywords: nanosuspension; laser fragmentation; megestrol acetate; preclinical development; oral bioavailability 


\section{Introduction}

An important fraction ( $40 \%)$ of the new drug candidates emerging from drug discovery programs has poor water solubility and this trend is not expected to change in the future $[1,2]$. The aqueous solubility of a drug is a prime determinant of its dissolution rate. Incomplete oral bioavailability occurs when the dissolution rate of a drug is so slow, that dissolution takes longer than the transit time past its absorptive sites [3, 4]. Poor aqueous solubility, is therefore becoming a major hurdle for formulation scientists working in the field of oral drug delivery [5]. Formulation approaches to tackle solubility and dissolution-related problems include the conversion of the hydrophobic drug to a salt, micellar solubilization, use of cosolvents, complexation of the drug (e.g. with cyclodextrins) and the molecular dispersion of the drug in a hydrophilic polymer matrix [6]. A simpler and more versatile strategy to improve dissolution is to reduce the particle size down to the nanometer size range, and thereby enlarge substantially the specific surface area $[2,5-7]$. Nanonization may be achieved either through precipitation of dissolved molecules (bottom-up approach) or comminution of larger particles (top-down approach). The precipitation process involves dissolving the drug in a solvent and precipitating it in a controlled manner to nanoparticles through mixing with an antisolvent $[5,8]$. Top-down methods for drug nanocrystal fabrication include media milling and high-pressure homogenization, two techniques that are used commercially. Media milling is currently the most common process with five of the six commercial products based on this technique [8]. In this method, a chamber is charged with milling media (typically beads of glass, zirconium oxide or highly cross-linked polystyrene resin), water, drug and stabilizer [9]. The size reduction of the drug is achieved through impaction of the milling media with the drug particles when the beads are rotated.

Recently, for the purpose of formulation development, there has been a marked interest to downscale the production of nanosuspensions, at the preformulation stage and during late discovery when drug availability is scarce [10,11]. At the early stage of development, less than $100 \mathrm{mg}$ of the compound is typically available [5]. Media milling performed in well plates or in low volume vials was downscaled to $10 \mathrm{mg}$ of drug compound. Wear or deformation of the wells may however limit its practicability [11]. Moreover, incomplete recovery of the drug from the milling media and inner milling chamber due to adhesion to the surfaces (a known potential shortcoming of the method [8]) could reduce the amount of the compound available for further experiments. The bottom-up liquid precipitation approach could also be adapted for the preparation of small quantities of drug nanosuspensions [12,13]. This method however requires that an appropriate pair of solvent/antisolvent is established and their ratio optimized for each compound, which is certainly a shortcoming in a tight drug supply context. Moreover, amorphous nanoparticles may result from this process and the organic solvents used should be eliminated from the formulation to avoid toxic effects [14].

Laser ablation and fragmentation in liquids are increasingly popular techniques for the rapid and simple production of inorganic and organic nanoparticles [15-17]. In laser ablation, a solid target is irradiated and the ejected material forms nanoparticles in the surrounding liquid. In laser fragmentation, a stirring suspension of microparticles is irradiated breaking them into nanoparticles. Previously, nanosecond (ns) or picoseconds (ps) laser fragmentation were used for the nanonization of organic materials such as phthalocyanines, quinadrone, buckminsterfullerene 
$\left(\mathrm{C}_{60}\right)$, 3,4,9,10-perylenetetracarboxylicdianhydride and melamine cyanurate [18-24]. Alternatively, femtosecond (fs) laser technology has been shown to generate smaller sized organic particles than ns-laser [25], and offers the advantage of irradiating materials with less energy, thus limiting the possible alteration of the ablated drugs nanocrystals. All the above studies were, however, not intended for applications in drug delivery and reported very little information on the degradation and on the polymorphic transformation of the organic material upon laser fragmentation. Recently, fs laser fragmentation was investigated for the nanonization of the anticancer agent paclitaxel. The method was found to be particularly well-suited for the treatment of small quantities of drug, therefore suggesting its application as a preclinical screening tool. When it is established that nanocrystal formulation is suitable and greater quantities of the lead compounds become available, than conventional techniques (such as media milling) may take the relay in the drug development process. Paclitaxel being a labile molecule and prone to polymorphic transformation in presence of water, the laser process was however accompanied by significant chemical degradation [26].

In this follow-up proof-of concept study, the fs and ns laser fragmentation were explored for the nanonization of minute amount of water-insoluble model drug megestrol acetate (MA) in the preclinical formulation development context. The influence of process parameters on the size and physicochemical properties of the drug was investigated in detail. MA nanocrystals were also prepared by media milling for comparison purposes. The in vitro dissolution kinetics of the nanocrystals were characterized and their oral pharmacokinetics assessed in rats.

\section{Materials and methods}

\subsection{Materials}

Megestrol acetate (>99\%), hydrocortisone 21-acetate (>98\%) and sodium dodecyl sulfate $(>98.5 \%)$ were purchased from Sigma-Aldrich (Oakville, ON, Canada). Poloxamer 188 was supplied by BASF (Mississauga, ON, Canada). Zirconium oxide (yttrium oxide stabilized) grinding spheres with a diameter of $0.8 \mathrm{~mm}$ were purchased from Glenmills (Clifton, NJ). All aqueous solutions were prepared using deionised water $(18.2 \mathrm{M} \Omega \cdot \mathrm{cm})$ generated with a Millipore Milli-Q system (Bedford, MA). The water, acetonitrile and methanol used for LC-MS analyses were all HPLC grade and purchased from JT Baker (Phillipsburg, NJ). The ethyl acetate (ACS reagent) and 2-propanol (CMOS grade) were from JT Baker (Phillipsburg, NJ). The formic acid had a purity of 98-100\% and was purchased from AnalaR EMD (Darmstadt, Germany).

\subsection{Laser fragmentation}

MA suspensions $(0.5 \mathrm{mg} / \mathrm{mL})$ were prepared by adding micrometric powder to water, followed by $30 \mathrm{~min}$ of sonication and a further 30 -min period of vigorous magnetic stirring. Laser fragmentation was obtained by focusing a laser radiation on a magnetically stirred suspension of the drug. Two types of laser radiations were investigated: a fs radiation (120 fs, $800 \mathrm{~nm}, 1 \mathrm{kHz}$, Ti:sapphire Hurricane laser, Newport Corporation, Mountain View, CA) at powers ranging from 50 to $400 \mathrm{~mW}$, and a ns radiation $(5 \mathrm{~ns}, 532 \mathrm{~nm}, 10 \mathrm{~Hz}, \mathrm{Nd}$ :YAG Brilliant B laser, Quantel, Paris, France) at powers ranging from 500 to $2500 \mathrm{~mW}$. In both cases, the beam was focused by a lens with a focal length of $10 \mathrm{~cm}$. The fs laser fragmentation was performed using a vertical 
configuration (Fig. 1a) with the focus located $\sim 3 \mathrm{~mm}$ below the surface of the liquid. The effects of treatment time and volume of suspension were studied. In the case of ns laser fragmentation, the horizontal configuration with the laser focused in the middle of the suspension (Fig. 1b) was used in order to avoid splashing of the liquid that may occur when the vertical configuration is used at high laser powers. For the in vitro and in vivo studies, a fs laser fragmented MA suspension was prepared $(250 \mathrm{~mW}$ for $30 \mathrm{~min})$ in $2 \mathrm{~mL}$ batches as described above except that the drug concentration was $0.65 \mathrm{mg} / \mathrm{mL}$ in freshly prepared poloxamer $188(8 \mathrm{mg} / \mathrm{mL})$ aqueous solution. The drug concentration was adjusted to $0.5 \mathrm{mg} / \mathrm{mL}$ prior to the in vitro and in vivo experiments.
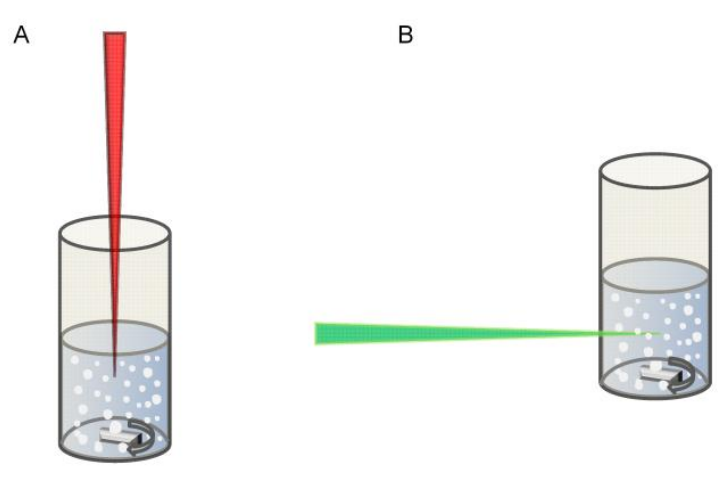

Fig. 1. Representation of laser fragmentation in (A) the vertical and (B) the horizontal configurations.

\subsection{Preparation of nanosuspensions by media milling}

A nanoparticulate MA dispersion was prepared using a roller mill. A 30-mL glass bottle was charged with $10 \mathrm{~mL}$ of $0.8 \mathrm{~mm}$ diameter zirconium oxide beads, $150 \mathrm{mg}$ of MA and $7.5 \mathrm{~mL}$ of an aqueous solution of poloxamer $188(0.8 \% \mathrm{w} / \mathrm{v})$. The bottle was rolled at $70 \mathrm{rpm}$ for $72 \mathrm{~h}$. The resulting nanosuspension was separated from the beads using a centrifuge filter with a $10 \mu \mathrm{m}$ mesh (VectaSpin 20, Whatman, Piscataway, NJ) and further syringe-filtered through a 1.0- $\mu \mathrm{m}$ mesh to remove any micrometric particles. The drug concentration was adjusted to $0.5 \mathrm{mg} / \mathrm{mL}$ with a solution of poloxamer $188(0.8 \% \mathrm{w} / \mathrm{v})$.

\subsection{Evaluation and identification of the chemical degradation}

High performance liquid chromatography was used to evaluate the chemical composition of the laser fragmented samples. Prior analysis, the MA suspensions were first mixed with equal volume of acetonitrile in order to dissolve the drug particles. Separations were performed on an 1100 LC system coupled to an ESI-MSD-TOF mass spectrometer (Agilent Technologies, Santa Clara, CA). The chromatographic column was a Gemini C6-Phenyl, 150 x $4.6 \mathrm{~mm}, 5 \mu \mathrm{m}$ particle size (Phenomenex , Torrance, CA) operated at $0.5 \mathrm{~mL} / \mathrm{min}$. The eluents were $0.1 \%(v / v)$ formic acid in water and $0.1 \%(\mathrm{v} / \mathrm{v})$ formic acid in acetonitrile. A two step acetonitrile linear gradient was used for elution. After the injection of $2 \mu \mathrm{L}$ of sample, the acetonitrile concentration was increased from 70 to $80 \%$ over 11 min then from 80 to $90 \%$ over the next minute. A 1 -min flush time at $90 \%$ was followed by an equilibration step with the initial mobile phase 
composition for a total run time of $19 \mathrm{~min}$. Both UV and mass spectrometric detections were used. The UV signal was acquired at $254 \mathrm{~nm}$ and the chromatograms were used to quantify the MA in the samples and evaluate chemical degradation (chromatographic purity). The relative intensities of the area under the curve of the impurity peaks in comparison to the MA peak area found in the chromatograms were used to estimate the percentages of chemical degradation in the laser treated samples. The mass spectrometer was operated in positive electrospray mode with a dual spray configuration allowing for internal calibration and therefore a very good mass accuracy. This permitted the determination of empirical formulas for degradation products. Mass spectra were acquired from $\mathrm{m} / \mathrm{z}, 50$ to 2000 with an acquisition cycle of $0.97 \mathrm{~s}$ and a resolution greater than 10000 . The electrospray voltage was set at $4 \mathrm{kV}$, the fragmentor at $140 \mathrm{~V}$ and the source temperature at $350^{\circ} \mathrm{C}$.

\subsection{Evaluation of the size and morphology of the particles}

The average size of nanocrystals and the polydispersity index (PDI) were determined by dynamic light scattering (DLS) using a Malvern Zetasizer NanoSeries instrument (Malvern, Worcestershire, UK). Each measurement was performed on the undiluted suspension in low volume disposable sizing cuvette at $25^{\circ} \mathrm{C}$ in triplicate. The CONTIN algorithm was used to extract size distributions from the autocorrelation functions. The volume size distributions were obtained by laser diffraction (LD) using the Horiba LA-950 particle size distribution analyzer (Horiba Instruments, Inc., Kyoto, Japan) equipped with a MiniFlow circulation system. The dispersant was water and the complex refractive index used for MA particles was $1.55-i 0.1$.

Morphological evaluation of the MA particles after laser fragmentation in water was conducted by scanning electron microscopy (SEM). A drop of suspension was placed on a silicon substrate and dried overnight in a dessicator under vacuum. The sample was observed with a FE-MEB S4700 field-emission scanning electron microscope (Hitachi, Tokyo, Japan). An untreated MA control sample (water-exposed non-fragmented) was also observed for comparison. Some samples were also studied by transmission electron microscopy (TEM) and the methodology is described in the Supporting Material.

\subsection{Physicochemical characterization}

MA samples prepared in water were analyzed by elemental analysis, Fourier transform infrared spectroscopy (FTIR) and X-ray photoelectron spectroscopy (XPS) after $72 \mathrm{~h}$ of lyophilization to evaluate their chemical integrity after laser treatment. The elemental analysis was performed on an ECS 4010 elemental analyzer (Costech, Valencia, CA). The 'insoluble isolated degradation' sample consisted of a fs laser fragmented $(250 \mathrm{~mW}, 30 \mathrm{~min}$ in $2 \mathrm{~mL}$ vials $)$ sample batch dialysed (regenerated cellulose membrane with cut-off MW 6-8000, Spectrum Laboratories Inc., Rancho Dominguez, CA) for $48 \mathrm{~h}$ against 30\% ( $/ / v)$ 2-propanol in water, followed by further $24 \mathrm{~h}$ dialysis against water. The content of the dialysis bag was lyophilized for $72 \mathrm{~h}$ prior analysis. About $2 \mathrm{mg}$ of the sample (except for the 'isolate degradation' sample where only $0.5 \mathrm{mg}$ was available) was weighed precisely in a tin pan and the mass elemental composition of $\mathrm{C}$ and $\mathrm{H}$ were determined. The difference between $100 \%$ and the sum of the percentages found for $\mathrm{C}$ and $\mathrm{H}$ was attributed to $\mathrm{O}\left(\mathrm{O}_{\% w / w}=100 \%-\left(\mathrm{C}_{\% w / w}+\mathrm{H}_{\% w / w}\right)\right)$. FTIR analysis, in the range 400-4000 $\mathrm{cm}^{-1}$ with a resolution of $4 \mathrm{~cm}^{-1}$, was performed with a Bio-Rad Excalibur Series spectrometer, 
FTS3000 (Bio-rad Laboratories, Randolph, MA) using the potassium bromide (KBr) pellet technique. Each $150 \mathrm{mg}$ pellet contained $2 \mathrm{mg}$ of the MA sample. The surface chemistry study performed with XPS is described in the Supporting Material. The crystallinity of the laser treated samples was evaluated with X-ray diffraction (XRD) and differential scanning calorimetry (DSC). Prior to the analysis, the samples suspended in water were lyophilized for $72 \mathrm{~h}$ and the samples suspended in poloxamer 188 were cleaned by ultracentrifugation (4 cycles of centrifugation at 29,400 x $g$ for 20 min followed by redispersion in deionized water), and then lyophilized. XRD spectra were obtained with an X'Pert X-ray PANAlytical diffractometer (PANalytical Inc, Montreal, QC, Canada) using the grazing angle method in which the X-ray source was fixed at $\omega=2^{\circ}$. Samples of $10-15 \mathrm{mg}$ were used. The X-ray source was $\mathrm{CuK} \alpha(50 \mathrm{kV}$ $40 \mathrm{~mA}$ ). The $2 \theta$ range scanned was 5 to $35^{\circ}$ at a rate of $0.02^{\circ} 2 \theta / \mathrm{s}$. Thermal properties were obtained by DSC (DSC 7, Perkin Elmer, Waltham, MA) at a heating rate of $5^{\circ} \mathrm{C} / \mathrm{min}$. Samples of 2-3 mg were weighed, sealed in aluminum pans and analyzed under an helium purge of 25 $\mathrm{mL} / \mathrm{min}$. The instrument was previously calibrated with respect to temperature and enthalpy using an indium standard.

\subsection{In vitro dissolution study}

Dissolution studies were carried out using a dissolution apparatus (Distek 2100A, North Brunswick, NJ) following the USP Apparatus II (paddle) method. The paddle speed and bath temperature were set at $50 \mathrm{rpm}$ and $37 \pm 1^{\circ} \mathrm{C}$, respectively. The dissolution medium consisted of $900 \mathrm{~mL} 0.5 \%(w / v)$ SDS aqueous solution and provided sink conditions. Three suspensions containing $0.5 \mathrm{mg} / \mathrm{mL}$ of the drug prepared in a solution of poloxamer $188(8 \mathrm{mg} / \mathrm{mL})$ were tested: 1) a suspension of the coarse (untreated) MA; 2) a MA nanosuspension prepared by media milling; and 3) a MA suspension prepared by fs laser fragmentation $(250 \mathrm{~mW}, 30 \mathrm{~min})$ in batches of $2 \mathrm{~mL}$. A 5-mL sample of the MA formulations was added into the dissolution medium. A 2-mL aliquot was taken at specific time intervals and immediately filtered through a $0.02-\mu \mathrm{m}$ syringe filter. The drug concentration was determined based on the absorbance value read by a UV-vis spectrophotometer (Cary 100 Bio, Varian, Palo Alto, CA) at $292 \mathrm{~nm}$. Each experiment was performed in triplicate.

\subsection{Pharmacokinetic study}

All animal studies were conducted as approved by the Animal Welfare and Ethics Committee of the University of Montreal in accordance with the Canadian Council on Animal Care guidelines. Male Srague-Dawley rats weighing 270-300 g (Charles River, Montreal, QC, Canada) were assigned to 3 treatment groups of 6 animals/group. The animals had free access to food and water before and during the experiment. Three suspensions containing $0.5 \mathrm{mg} / \mathrm{mL}$ of the drug were prepared in a solution of poloxamer $188(8 \mathrm{mg} / \mathrm{mL}): 1)$ a suspension of the coarse (untreated) MA as a negative control; 2) a MA nanosuspension prepared by media milling as a positive control; and 3) a MA suspension prepared by fs laser fragmentation ( $250 \mathrm{~mW}, 30 \mathrm{~min})$ in batches of $2 \mathrm{~mL}$. The MA formulations were administered orally by gavage at a dose of 5 $\mathrm{mg} / \mathrm{kg}$. Blood samples $(300 \mu \mathrm{L})$ were collected from the subclavian vein under isoflurane anesthesia in EDTA coated tubes prior to the administration of the drug and after 30 min, 1, 2, 3, 4, 6, 8, 10 and $24 \mathrm{~h}$. The plasma was separated by centrifugation at $2000 \mathrm{x} \mathrm{g}$ for $10 \mathrm{~min}$. A 100 $\mu \mathrm{L}$ aliquot of plasma was transferred to an Eppendorf tube, and hydrocortisone acetate was 
added as an internal standard (at a concentration of $100 \mathrm{ng} / \mathrm{mL}$ ). Following liquid-liquid extraction with $500 \mu \mathrm{L}$ of ethyl acetate and reconstitution of the residue with $100 \mu \mathrm{L}$ of methanol-water $(70: 30, v / v)$, the concentration of MA was quantified by LC-MS/MS.

Separations were performed on a Surveyor LC system coupled to a Quantum Ultra AM triple quadrupole mass spectrometer (all from Thermo Fisher, San Jose, CA). The chromatographic column was a Gemini C6-Phenyl, 50 x $4.6 \mathrm{~mm}, 3 \mu \mathrm{m}$ particle size (Phenomenex, Torrance, CA) operated at $0.5 \mathrm{~mL} / \mathrm{min}$. After the injection of $10 \mu \mathrm{L}$ of the sample, a linear gradient elution, using $0.1 \%(v / v)$ formic acid in water (mobile phase A) and $0.1 \%(v / v)$ formic acid in methanol (mobile phase B) was performed by starting at $68 \% \mathrm{~B}$ and increasing to $75 \% \mathrm{~B}$ over 5 min. A constant $75 \%$ B was held for an additional 1 min followed by an equilibration step with the initial mobile phase for a total run time of $10 \mathrm{~min}$. Positive ions were generated using an atmospheric pressure chemical ionization source (APCI). Mass spectra were acquired in SRM mode with the transition $405.3 \rightarrow 309.2$ used for the internal standard (hydrocortisone acetate) and $385.2 \rightarrow 267.1$ and $325.2 \rightarrow 267.1$ used for MA. Scan time was 1 s for each SRM transition and the two transitions of the target compound were summed together in data analysis process. Collision energy and tube lens were optimized before each analysis. Their values were comprised between 15 and $20 \mathrm{~V}$ and 100 to $110 \mathrm{~V}$, respectively. A $4 \mu \mathrm{A}$ current was applied to the corona needle and the capillary temperature was set at $300^{\circ} \mathrm{C}$. Calibration samples were prepared by spiking $400 \mu \mathrm{L}$ of rat plasma with $10 \mu \mathrm{L}$ MA solution in methanol and $10 \mu \mathrm{L}$ aliquots were injected into the LC-MS/MS system. The calibration curve was found to be linear from 5 to $1000 \mathrm{ng} / \mathrm{mL}$ with a correlation coefficient $\mathrm{r}^{2}=0.998$. The pharmacokinetic parameters for MA were calculated using non-compartmental analysis and were compared with ANOVA followed by Tuckey's multiple comparison post-hoc test. Data manipulation and statistics were performed using Graph Pad Prism V.4.00 (Graph Pad Software Inc., San Diego, CA). The level of statistical significance was fixed at $p<0.05$.

\section{Results and discussion}

\section{Laser fragmentation study}

In this part of the study, laser fragmentation experiments were performed in the absence of stabilizers in order to evaluate directly the effects of fabrication parameters and simplify interpretation of the characterization data. The effects of laser power, suspension volume and treatment time were explored during fs laser fragmentation of MA in deionized water. The average particle diameter and PDI measured by DLS and the chemical degradation percentage estimated by liquid chromatography, after dissolution of the drug particles with the addition of methanol, are presented in Table 1. The initial untreated MA suspension had a size of $3100 \pm$ $600 \mathrm{~nm}(\mathrm{PDI}=0.6 \pm 0.3)$ and contained $0.6 \pm 0.2 \%$ of impurities. Independently of suspension volume and treatment time, as the laser power increased, average particle size (and PDI) decreased, but at a cost of higher degradation. The same trend had been observed during the fs laser fragmentation of paclitaxel, although to a greater extent [26]. For a given suspension volume/ablation time ratio ( $60 \mathrm{~min}$ in $10 \mathrm{~mL} v s .12 \mathrm{~min}$ in $2 \mathrm{~mL}$ ), the smaller volume resulted in smaller particles, possibly due to a more uniform mixing during treatment. Increasing the duration of the treatment further reduced the particle size. 
Table 1. Particle diameter, PDI and chemical degradation (Deg) of MA suspensions prepared by fs laser fragmentation in water. Mean \pm SD $(n=3-6)$.

\begin{tabular}{|c|c|c|c|c|c|c|c|c|c|}
\hline \multirow[b]{2}{*}{ Power (mW) } & \multicolumn{3}{|c|}{$10 \mathrm{~mL}, 60 \mathrm{~min}$} & \multicolumn{3}{|c|}{$2 \mathrm{~mL}, 12 \mathrm{~min}$} & \multicolumn{3}{|c|}{$2 \mathrm{~mL}, 30 \mathrm{~min}$} \\
\hline & Size (nm) & PDI & $\operatorname{Deg}(\%)$ & Size (nm) & PDI & $\operatorname{Deg}(\%)$ & Size (nm) & PDI & $\operatorname{Deg}(\%)$ \\
\hline 50 & $1840 \pm 430$ & $0.8 \pm 0.2$ & $0.5 \pm 0.2$ & $1810 \pm 400$ & $0.9 \pm 0.2$ & $0.5 \pm 0.1$ & $1490 \pm 300$ & $0.5 \pm 0.2$ & $1.2 \pm 0.3$ \\
\hline 100 & $1600 \pm 300$ & $0.7 \pm 0.3$ & $0.8 \pm 0.5$ & $1300 \pm 220$ & $0.4 \pm 0.1$ & $1.4 \pm 0.7$ & $1170 \pm 220$ & $0.4 \pm 0.2$ & $2.2 \pm 0.2$ \\
\hline 250 & $1160 \pm 200$ & $0.3 \pm 0.1$ & $1.5 \pm 0.5$ & $960 \pm 130$ & $0.3 \pm 0.1$ & $1.7 \pm 0.1$ & $800 \pm 140$ & $0.2 \pm 0.1$ & $2.8 \pm 0.8$ \\
\hline 400 & $900 \pm 70$ & $0.3 \pm 0.1$ & $2.4 \pm 0.7$ & $810 \pm 70$ & $0.3 \pm 0.1$ & $2.6 \pm 0.6$ & $640 \pm 80$ & $0.2 \pm 0.1$ & $3.6 \pm 1.0$ \\
\hline
\end{tabular}

The nature of the degradation products induced during fs laser fragmentation was investigated with mass spectrometry (Table S1). The empirical molecular formulas for the degradation products found in the samples suggested that oxidation through addition of oxygen or elimination of hydrogen, as well as scission of the acetate group were the most likely to occur. An important fraction of these degradation products, i.e. $80 \pm 10 \%$, was dissolved in the aqueous media and could be removed from the suspension with a centrifugation step $(17,000 \times \mathrm{x}, 10$ min). Two additional centrifugation steps did not result in further reduction of the impurities levels, suggesting that part of the degradation products was present at the particle level. It is noteworthy that the MA suspension exhibited a beige tint after fs laser treatment. It was found that this colour originated from nanoparticles that were insoluble in water (and other organic solvents such as methanol, acetone and 2-propanol) which could be isolated by dialysis against a mixture of isopropanol and water (allowing for the elimination of MA). In the case of a suspension prepared by fs laser fragmentation at $250 \mathrm{~mW}$ for $30 \mathrm{~min}$ in $2 \mathrm{~mL}$ batches, a brown powder representing $\sim 7 \%$ of the initial drug mass was obtained after lyophilisation of the dialysis membrane content.

The ns laser fragmentation at a wavelength of $532 \mathrm{~nm}$ was also evaluated at powers ranging from 500 to $2500 \mathrm{~mW}$ for $10 \mathrm{~mL}$ suspensions treated for $60 \mathrm{~min}$. As observed for fs laser fragmentation, particle size decreased as laser power was increased, again at a cost of higher degradation. Compared to fs laser, the degradation induced during ns laser treatment was more important for a given particle size achieved. The same degradation products found by mass spectrometry in the fs laser fragmented samples could be identified in the ns laser treated suspensions. Although the beige tint was less apparent after ns laser fragmentation, the content in MA measured by liquid chromatography of an accurately weighed sample of the lyophilized drug powder after ns laser fragmentation $(2500 \mathrm{~mW}, 1 \mathrm{~h}, 10 \mathrm{~mL})$ indicated a mass loss of $20 \%$ of the drug. A value significantly higher than the $5.3 \%$ degradation estimated by evaluation of the chromatographic purity, and suggesting the presence of an insoluble degradation product similar to that found after fs laser fragmentation. 
Table 2. Particle diameter, PDI and chemical degradation (deg) of MA suspensions prepared by ns laser fragmentation $(10 \mathrm{~mL}, 60 \mathrm{~min})$ in water. Mean $\pm \mathrm{SD}(\mathrm{n}=3-7)$.

\begin{tabular}{cccc}
\hline Power $(\mathbf{m W})$ & Size $(\mathbf{n m})$ & PDI & Deg $(\%)$ \\
\hline $\mathbf{5 0 0}$ & $1430 \pm 180$ & $0.5 \pm 0.1$ & $1.3 \pm 0.2$ \\
$\mathbf{1 0 0 0}$ & $1140 \pm 210$ & $0.5 \pm 0.1$ & $2.6 \pm 0.7$ \\
$\mathbf{2 0 0 0}$ & $960 \pm 170$ & $0.5 \pm 0.1$ & $3.9 \pm 1.1$ \\
$\mathbf{2 5 0 0}$ & $830 \pm 110$ & $0.5 \pm 0.1$ & $5.3 \pm 1.5$ \\
\hline
\end{tabular}

The size assessments by SEM and LD confirmed that significant nanonization occured during fs (250 mW, $30 \mathrm{~min}, 2 \mathrm{~mL}$ ) and ns laser fragmentation (2.5 W, $60 \mathrm{~min}, 10 \mathrm{~mL}$ ) (Fig. 2). Indeed, $\sim 30 \%$ and $\sim 60 \%$ of the drug mass was $<1 \mu \mathrm{m}$ after the ns and fs laser treatments, respectively. In contrast, when milder fragmentation conditions were used (e.g. fs laser at $250 \mathrm{~mW}$ for $60 \mathrm{~min}$ in $10 \mathrm{~mL}$ and $\mathrm{ns}$ laser at $1 \mathrm{~W}$ for $60 \mathrm{~min}$ in $10 \mathrm{~mL}$ ) mostly micronization occurred with the majority of particles having a diameter in the 1-10 $\mu \mathrm{m}$ range after the laser treatment (data not shown). Furthermore, the total degradation (estimated by the quantification of MA from accurately weighed lyophilized samples) after micronization of the drug powder remained within $1 \%$ of the values estimated by the impurity peak areas of the chromatograms, meaning that the production of insoluble degradation products was much less important in these conditions. 

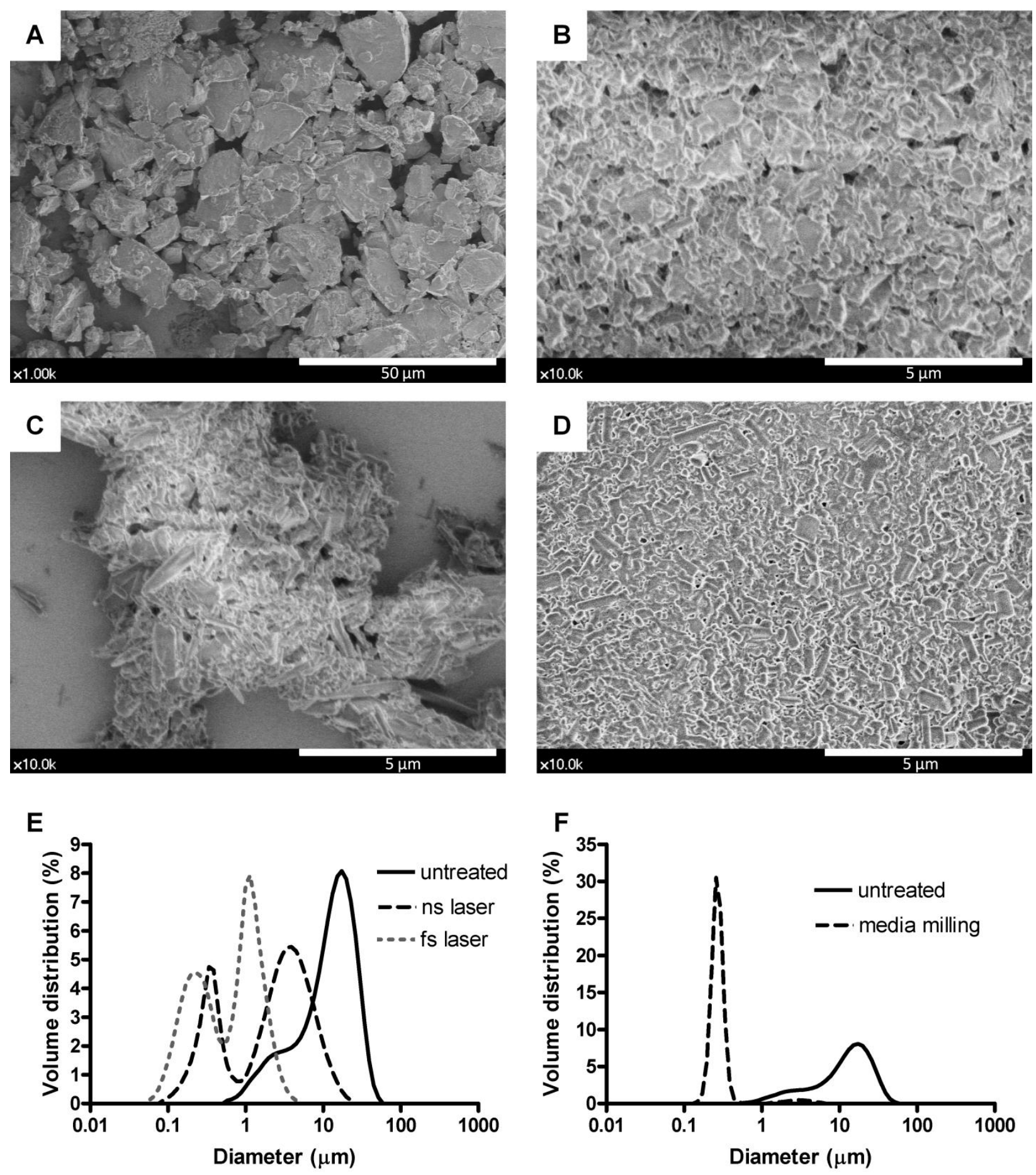

Fig. 2. Size analysis of MA particles. Scanning electron micrographs of (A) untreated waterexposed MA; (B) fs laser fragmented MA (250 mW, $30 \mathrm{~min}, 2 \mathrm{~mL}$ ); (C) ns laser fragmented MA (2.5 W, $60 \mathrm{~min}, 10 \mathrm{~mL}$ ); and (D) media milled MA. Size distribution analysis by laser diffraction of $(\mathrm{E})$ the laser fragmented MA samples and $(\mathrm{F})$ the media milled MA compared to the untreated drug. 


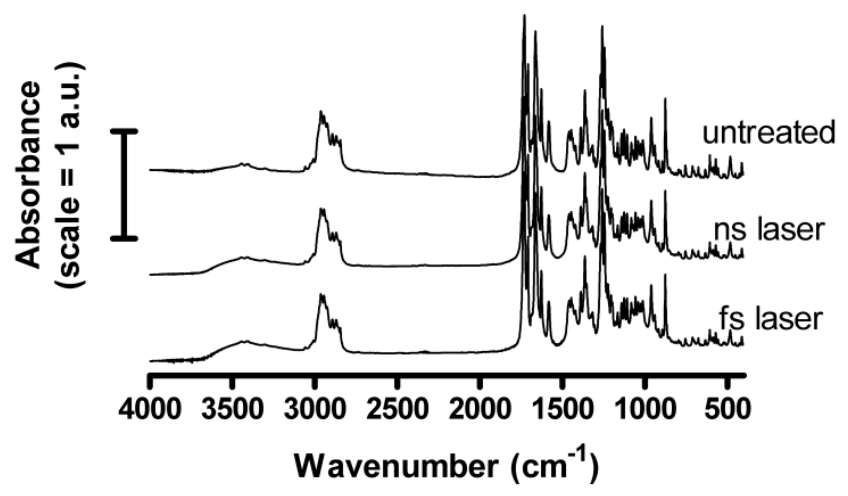

Fig. 3. FTIR spectra of water exposed untreated MA (untreated), ns laser (2.5 W, 60 min, 10 $\mathrm{mL}$ ) fragmented MA (ns laser) and fs laser (250 mW, $30 \mathrm{~min}, 2 \mathrm{~mL}$ ) fragmented MA (fs laser).

FTIR was employed to evaluate the effect of laser fragmentation on the chemical integrity of the drug. The fingerprint region of the spectra $\left(2000\right.$ to $\left.400 \mathrm{~cm}^{-1}\right)$ did not vary with laser treatment. The only clear difference between laser treated and untreated samples was an increase of the $(\mathrm{OH})$ band at $\sim 3440 \mathrm{~cm}^{-1}$ suggesting that oxidation occurred. Further evaluation of the chemical composition of the lyophilized laser-treated drug powder was performed with elemental analysis (Table 3). The composition of the initial MA powder was within the experimental error $(0.3 \%)$ of the expected (theoretical) values for this drug. An increase in oxygen mass percentages and oxygen/carbon ratios occurred after laser treatment, suggesting partial oxidative degradation of MA. It is interesting to note that the pre-laser ultrasound and stirring steps (MA suspension control) already participated to the oxidation process. The fact that the hydrogen/carbon ratio remained constant in all samples suggested that the oxidative degradation principally consisted in the substitution of hydrogen by hydroxyl groups.

Table 3. Atomic composition (mass percentages) and ratios of untreated and laser fragmented MA revealed by elemental analysis.

\begin{tabular}{lccccc}
\hline \multicolumn{1}{c}{ Sample } & $\mathbf{C}(\boldsymbol{\%})$ & $\mathbf{H}(\boldsymbol{\%})$ & $\mathbf{O}(\boldsymbol{\%})$ & $\mathbf{H} / \mathbf{C}$ & $\mathbf{O} / \mathbf{C}$ \\
\hline MA theoretical & 74.9 & 8.3 & 16.8 & 0.11 & 0.22 \\
MA powder & 74.8 & 8.4 & 16.8 & 0.11 & 0.22 \\
MA suspension control & 73.8 & 8.4 & 17.8 & 0.11 & 0.24 \\
ns laser 2500 $\mathbf{~ W W ~} \mathbf{1} \mathbf{~ h ~} \mathbf{1 0} \mathbf{~ m L}$ & 72.8 & 8.2 & 19.0 & 0.11 & 0.26 \\
fs laser 250 $\mathbf{~ W W ~} \mathbf{1} \mathbf{~ h ~} \mathbf{1 0} \mathbf{~ m L}$ & 73.3 & 8.0 & 18.7 & 0.11 & 0.26 \\
fs laser 250 $\mathbf{~ m W ~ 3 0 ~} \mathbf{~ m i n ~} \mathbf{~} \mathbf{~ m L}$ & 68.1 & 7.6 & 24.3 & 0.11 & 0.36 \\
Isolated degradation & 51.1 & 4.7 & 44.2 & 0.09 & 0.86 \\
\hline
\end{tabular}

An XPS study was also conducted in order to gain insight on the surface chemistry of the MA particles. The $\mathrm{C} 1 \mathrm{~s}$ high resolution XPS spectra were deconvoluted with 4 peaks corresponding to the following binding energies: $\mathrm{C}=\mathrm{C} / \mathrm{C}-\mathrm{C}$ at $285 \mathrm{eV}$; $\mathrm{C}-\mathrm{O}$ at $286.6 \mathrm{eV} ; \mathrm{C}=\mathrm{O}$ at $288 \mathrm{eV}$ and $\mathrm{O}-$ $\mathrm{C}=\mathrm{O}$ at $289.3 \mathrm{eV}$. The contribution of the $\mathrm{C}-\mathrm{O}$ bond clearly increased after laser treatment (Table S2), a further confirmation that partial oxidative degradation of the drug occurred most probably 
through addition of hydroxyl groups. This more hydrophilic surface could explain why stable nanosuspensions were obtained without the addition of stabilizing agent with laser fragmentation in water. The insoluble degradation product isolated by dialysis (insoluble isolated degradation) after fs laser fragmentation $(250 \mathrm{~mW}, 30 \mathrm{~min}, 2 \mathrm{~mL})$ was also analyzed by elemental analysis (Table 3) and XPS (Table S2), and both methods showed that MA had suffered oxidative degradation to transform into this brown organic compound. The exact mechanism by which the degradation occurred is unknown. The high local temperature generated at the focal point during laser fragmentation is a possible cause.

The crystallinity of the drug powder following laser fragmentation was investigated with XRD and DSC. The crystal phase of the drug was conserved after laser treatment (Fig. 4). The DSC analysis (Table 3 and Fig. S1), however, indicated that partial loss in crystallinity of the drug occurred during laser fragmentation as suggested by a reduction of the melting enthalpy peak.

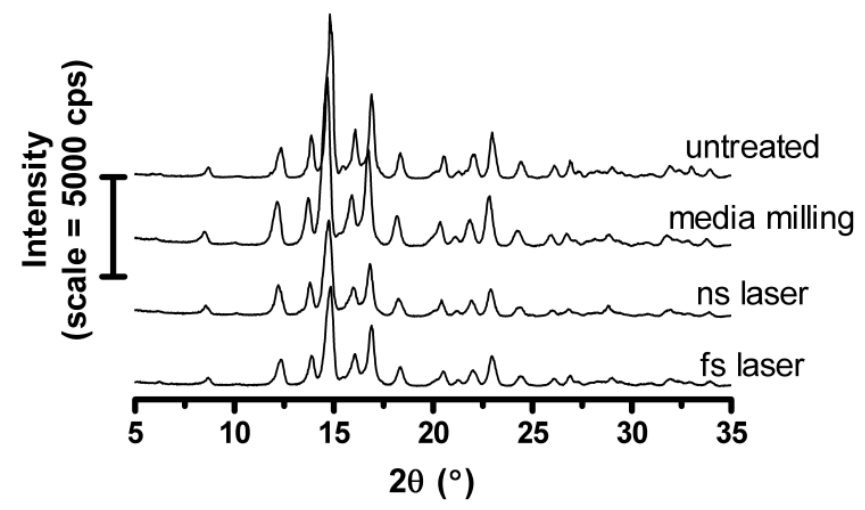

Fig. 4. XRD analysis of water exposed untreated MA (untreated), media milled (72 h) MA (media milling), ns laser (2.5 W, $60 \mathrm{~min}, 10 \mathrm{~mL})$ fragmented MA (ns laser) and fs laser (250

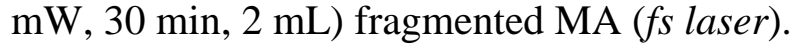

Table 5. Melting onset temperature and melting enthalpy of untreated, laser fragmented and media milled MA samples determined from DSC measurements. The percentages of crystallinity presented are estimated from the ratio of the melting enthalpies relatively to the untreated MA powder. Mean \pm SD $(\mathrm{n}=2-9)$.

\begin{tabular}{lccc}
\hline \multicolumn{1}{c}{ Sample } & $\begin{array}{c}\text { Onset temperature } \\
\left({ }^{\circ} \mathbf{C}\right)\end{array}$ & $\begin{array}{c}\text { Melting enthalpy } \\
(\mathbf{J} / \mathbf{g})\end{array}$ & $\begin{array}{c}\text { Crystallinity } \\
(\boldsymbol{\%})\end{array}$ \\
\hline MA powder untreated & $217.6 \pm 0.2$ & $82.9 \pm 1.7$ & 100 \\
ns laser, 2500 $\mathbf{~} \mathbf{W W} \mathbf{1} \mathbf{~ h , ~ 1 0 ~} \mathbf{~ m L}$ & $205.8 \pm 1.6$ & $56.4 \pm 1.7$ & 68.0 \\
fs laser, 250 $\mathbf{~} \mathbf{1} \mathbf{1} \mathbf{~} \mathbf{1 0} \mathbf{~ m L}$ & $215.3 \pm 0.3$ & $75.5 \pm 1.5$ & 91.1 \\
fs laser, 250 $\mathbf{~} \mathbf{3 0} \mathbf{~} \mathbf{m i n}, \mathbf{2} \mathbf{~ m L}$ & $208.1 \pm 3.4$ & $55.8 \pm 1.2$ & 67.3 \\
Media milling 72 h & $215.6 \pm 0.1$ & $72.6 \pm 2.1$ & 87.6 \\
\hline
\end{tabular}

\section{Comparison with media milling}

A nanoparticulate suspension of MA was prepared by media milling in order to compare the physicochemical properties of the nanoparticles obtained after laser fragmentation. Although laser fragmentation could be performed from a suspension of the drug in deionized water, media milling required the inclusion of a stabilizing agent. After a $72 \mathrm{~h}$ milling period in a solution of 
$0.8 \%(w / v)$ poloxamer 188 , the average particle size measured by DLS was $620 \pm 40 \mathrm{~nm}(\mathrm{PDI}=$ $0.25 \pm 0.02$ ). The suspension was further filtered through a $1-\mu \mathrm{m}$ mesh in order to remove microparticles present in the suspension after the milling process (LD size analysis data not shown). Following the filtration step, a nanosuspension with an average diameter of $310 \pm 20 \mathrm{~nm}$ (PDI $=0.13 \pm 0.04$ ) was obtained. The impurity content determined by liquid chromatography was not different than the untreated MA powder $(0.6 \pm 0.2 \%)$. To allow for direct comparison, fs laser fragmentation in conditions $(250 \mathrm{~mW}, 30 \mathrm{~min}, 2 \mathrm{~mL})$ resulting in significant nanonization with reasonable degradation was performed in a solution of $0.8 \%(w / v)$ poloxamer 188 . The size and degradation obtained were respectively $680 \pm 50 \mathrm{~nm}(\mathrm{PDI}=0.25 \pm 0.05)$ and $4.7 \pm 0.7 \%$, similar to those obtained using the same laser conditions in deionized water. The crystallinity of the media milled and fs laser fragmented nanosuspensions were evaluated by XRD and DSC, after removing the excess poloxamer 188 by centrifugation and lyophilization. Inclusion of poloxamer 188 during the fs laser fragmentation process had no effect on the XRD pattern and the DSC results presented above using the same fragmentation parameters in deionized water. After media milling, the crystal phase of the drug was conserved (Fig. 4), and the loss in crystallinity estimated by DSC was less important than after laser fragmentation (Table 5). The morphology of the fs laser fragmented particles and the nanoparticles obtained by media milling was also evaluated with a transmission electron microscope equipped with an energy dispersion spectrometry (EDS) analyzer (Fig. S2). The surfaces of the laser treated particles were less regular than those of the milled particles, in agreement with the lower crystallinity determined by DSC for the former sample. Finally, it is interesting to note that nanometer-sized needles of the grinding media (yttrium stabilized zirconium oxide) were present in the milled sample (confirmed by EDS), although the amount was not quantified.

\section{In vitro dissolution study}

The dissolution profiles, in sink conditions, of the fs laser fragmented MA (250 mW, $30 \mathrm{~min}, 2$ $\mathrm{mL}$ ), the nanosized MA prepared by media milling and the coarse (untreated) MA were compared (Fig. 5). The nanosuspensions prepared by media milling and fs laser fragmentation had similar dissolution profiles and dissolved more rapidly than the untreated drug powder as expected [27]. Indeed, > 95\% of the drug had dissolved within $10 \mathrm{~min}$ for the two nanosuspensions, while $3 \mathrm{~h}$ where necessary for the coarse MA powder. Considering that laser fragmentation decreased the crystallinity of the drug, which may result in a faster dissolution rate [27], a dissolution study was also performed under non-sink conditions in order to compare the dissolution performances under discriminating conditions. In that case, although both nanosuspensions dissolved again more rapidly than the coarse MA powder, the nanosuspension prepared by media milling reached $100 \%$ of the drug's saturation concentration more rapidly than the fs laser fragmented nanosuspension (Fig. S3). It follows that the reduced crystallinity found in the fs laser fragmented MA did not significantly improved its dissolution kinetics. The smaller particle size and more uniform suspension achieved with media milling are plausible explanations for the improved dissolution observed under non-sink conditions. 


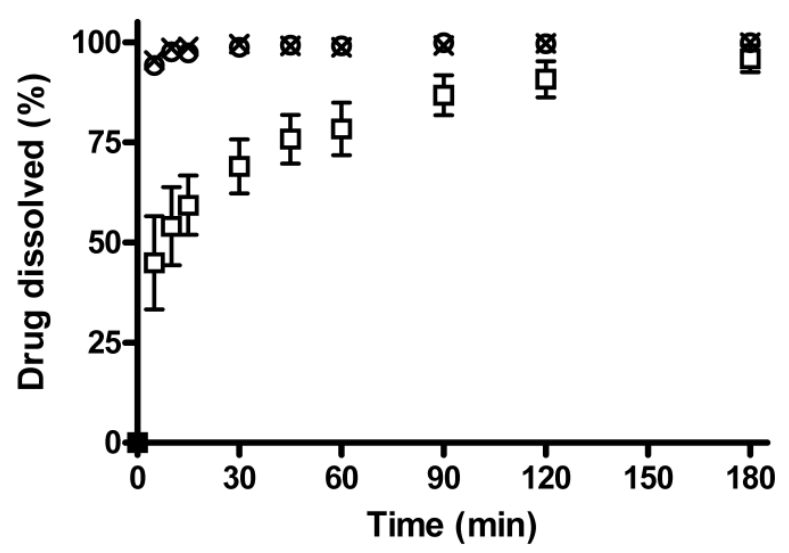

Fig. 5. Dissolution profiles in $900 \mathrm{~mL}$ of SDS $(0.5 \% \mathrm{w} / \mathrm{v})$ of $2.5 \mathrm{mg}$ MA from suspensions prepared from untreated MA powder (squares), media milled MA (crosses) and fs laser (250 $\mathrm{mW}, 30 \mathrm{~min}, 2 \mathrm{~mL})$ fragmented MA (circles) in a solution of poloxamer $188(0.8 \% \mathrm{w} / \mathrm{v})$. Mean $\pm \mathrm{SD}(\mathrm{n}=3)$.

\section{Bioavailability study}

The oral bioavailability of the fs laser fragmented MA (250 mW, $30 \mathrm{~min}, 2 \mathrm{~mL})$ was evaluated and compared with the nanosuspension of the drug prepared by media milling and a MA coarse (untreated) suspension. The results are shown in Fig. 6 and the relevant pharmacokinetic parameters are listed in Table 6. As expected, the relative bioavailablity of the nanosuspensions prepared by media milling and by fs laser fragmentation significantly increased compared to the coarse MA powder formulation. In both cases, nanonization more than doubled the $\mathrm{AUC}_{0-24 \mathrm{~h}}$ compared to the coarse powder, with the bioavailability of the fs laser fragmented formulation approaching (85\%) that of the media milled formulation. The maximum plasma concentration $\left(\mathrm{C}_{\mathrm{MAX}}\right)$ was higher for the fs laser fragmented formulation than for the two other groups. In this group, the highest concentration was reached in all rats at $0.5 \mathrm{~h}$ (first time point after drug administration), while it was more variable for the two other groups (1-4 $\mathrm{h}$ for the untreated MA and $0.5-3 \mathrm{~h}$ for the media milled MA), although the $\mathrm{T}_{\mathrm{MAX}}$ were not statistically different within groups. It is unclear why the fs laser fragmented formulation displayed a slightly different in vivo absorption profile than the media milled nanosuspension. Such behaviour could not be predicted from the in vitro dissolution study. The difference could lie in the chemical modifications of the drug particles surface induced by the laser treatment, although more experiments would be necessary to validate this hypothesis. 


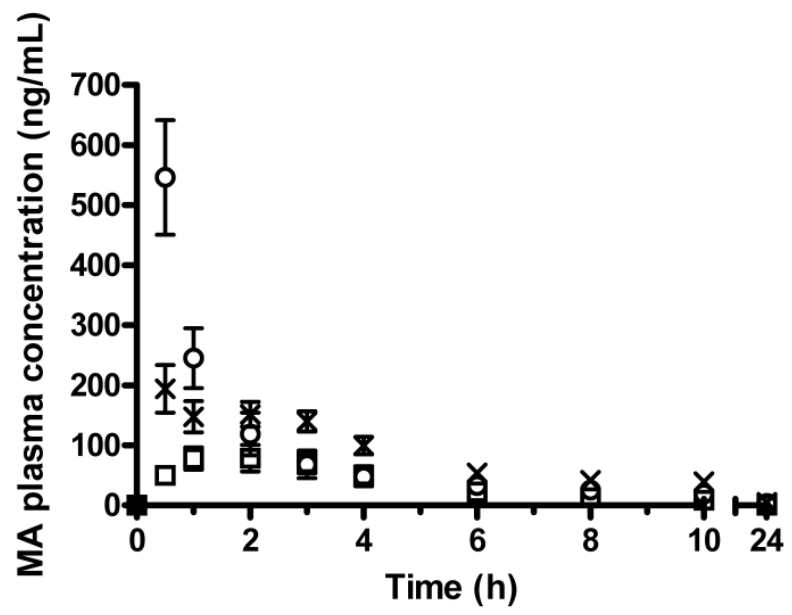

Fig. 6. Plasma concentration profiles of MA after oral administration of suspensions prepared from untreated MA powder (squares), media milled MA (crosses) and fs laser $(250 \mathrm{~mW}, 30 \mathrm{~min}$, $2 \mathrm{~mL}$ ) fragmented MA (circles) in a solution of poloxamer $188(0.8 \% \mathrm{w} / \mathrm{v})$ to Spraque-Dawley rats at a dose of $5 \mathrm{mg} / \mathrm{kg}$. Mean $\pm \operatorname{SEM}(\mathrm{n}=6)$.

Although laser fragmentation appears to be more detrimental to insoluble drugs than conventional milling methods, this study showed it could be used in screening tests to improve the in vivo bioavailability of MA using minute amount of the drug $(<15 \mathrm{mg}$ for the nanonization process). Once it is established that the use of a nanosuspension is beneficial for a specific drug, a conventional nanonization technique such as media milling could be applied for the production of greater amounts of nanocrystals with less degradation. Although drug degradation induced during laser fragmentation may be a concern (in particular for in vivo pharmacodynamic and toxicity studies), a significant fraction $(80 \pm 10 \%)$ could eventually be removed using a relatively simple centrifugation cleaning procedure. Furthermore, it can be expected that nanosuspensions with smaller particles, narrower size dispersions and less degradation may be generated in the future as the laser fragmentation methodology improves (e.g. use of a recirculation flow cell to better control the fabrication process [24, 28]). Furthermore, the technique in its current form could be useful for the micronization of minute amount of drugs (for potential application in pulmonary delivery [29]) as it would then generate less degradation products.

Table 6. Pharmacokinetic parameters after oral administration of suspensions prepared from untreated MA powder, media milled MA and fs laser (250 mW, $30 \mathrm{~min}, 2 \mathrm{~mL})$ fragmented MA to rats. Mean \pm SEM $(n=6)$.

\begin{tabular}{lccc}
\hline \multicolumn{1}{c}{ Parameter } & Untreated powder & Media milling & fs laser \\
\hline $\mathbf{C}_{\mathbf{M A X}}(\mathbf{n g} / \mathbf{m L})$ & $92 \pm 23$ & $213 \pm 35$ & $546 \pm 96^{* \pm}$ \\
$\mathbf{T}_{\mathbf{M A X}}(\mathbf{h})$ & $2.0 \pm 0.5$ & $1.6 \pm 0.5$ & 0.5 \\
$\mathbf{A U C}_{\mathbf{0 - 2 4 h}}(\mathbf{n g ~ h} / \mathbf{m L})$ & $446 \pm 116$ & $1188 \pm 131^{*}$ & $1005 \pm 174^{*}$ \\
\hline$* \mathrm{P}<0.05$ vs. untreated powder & & & \\
$£ \mathrm{P}<0.05$ vs. media milling & & &
\end{tabular}




\section{Conclusions}

In summary, laser fragmentation was explored for the fabrication of MA nanocrystals using minute amount of the drug in water or aqueous solution of a surfactant. Significant nanonization was achieved, although reduction in particle size was associated with increased degradation. Less degradation was induced when the fs laser radiation was used rather than the ns laser radiation to achieved a given size reduction. For both radiations, the degradation was primarily oxidative in nature. The original crystal phase of the drug was conserved after laser fragmentation, although a partial loss in crystallinity was observed. The in vitro dissolution profile and the in vivo bioavailability of the fs laser fragmented MA suspension were improved compared to a suspension of the coarse drug powder and were similar to a nanosuspension prepared by media milling. It follows that this proof-of-concept study supports the idea that this laser fragmentation technique could be used as preclinical screening tool for poorly water soluble compounds, in particular when drug availability is scarce. Work in progress is aiming at improving the laser technique to reduce size dispersion and chemical degradation. The optimization process will include the exploration of the formulation composition (e.g. nature of stabilizers) and the process parameters.

\section{Acknowledgements}

The authors thank F. Plourde for technical assistance with the in vivo study, S. Poulin for the XPS analyses, E. Nadezhina for the elemental analyses and J.-P. Masse for the TEM analyses. Financial support was provided by the Natural Sciences and Engineering Research Council of Canada (NSERC), the Canadian Institutes of Health Research (CIHR), the Groupe de Recherche Universitaire sur le Médicament (GRUM) and the Fonds de la recherche en santé du Quebec (FRSQ).

\section{References}

[1] S. Stegemann, F. Leveiller, D. Franchi, H. de Jong, H. Linden, When poor solubility becomes an issue: From early stage to proof of concept, Eur. J. Pharm. Sci., 31 (2007) 249-261.

[2] B.E. Rabinow, Nanosuspensions in drug delivery, Nat. Rev. Drug Discov., 3 (2004) 785-796. [3] D. Horter, J.B. Dressman, Influence of physicochemical properties on dissolution of drugs in the gastrointestinal tract, Adv. Drug Del. Rev., 46 (2001) 75-87.

[4] G.L. Amidon, H. Lennernäs, V.P. Shah, J.R. Crison, A Theoretical Basis for a

Biopharmaceutic Drug Classification: The Correlation of in Vitro Drug Product Dissolution and in Vivo Bioavailability, Pharm. Res., 12 (1995) 413-420.

[5] F. Kesisoglou, S. Panmai, Y. Wu, Nanosizing - Oral formulation development and biopharmaceutical evaluation, Adv. Drug Del. Rev., 59 (2007) 631-644.

[6] E. Merisko-Liversidge, G.G. Liversidge, E.R. Cooper, Nanosizing: a formulation approach for poorly-water-soluble compounds, Eur. J. Pharm. Sci., 18 (2003) 113-120.

[7] C.M. Keck, R.H. Muller, Drug nanocrystals of poorly soluble drugs produced by high pressure homogenisation, Eur. J. Pharm. Biopharm., 62 (2006) 3-16.

[8] H. Chen, C. Khemtong, X. Yang, X. Chang, J. Gao, Nanonization strategies for poorly watersoluble drugs, Drug Discov. Today, In Press, Corrected Proof. 
[9] A.A. Date, V.B. Patravale, Current strategies for engineering drug nanoparticles, Curr. Opin. Colloid Interface Sci., 9 (2004) 222-235.

[10] B. Van Eerdenbrugh, G. Van den Mooter, P. Augustijns, Top-down production of drug nanocrystals: nanosuspension stabilization, miniaturization and transformation into solid products, Int. J. Pharm., 364 (2008) 64-75.

[11] B. Van Eerdenbrugh, B. Stuyven, L. Froyen, J. Van Humbeeck, J.A. Martens, P. Augustijns, G. Van den Mooter, Downscaling drug nanosuspension production: processing aspects and physicochemical characterization, AAPS PharmSciTech, 10 (2009) 44-53.

[12] H. Zhao, J.-X. Wang, Q.-A. Wang, J.-F. Chen, J. Yun, Controlled Liquid Antisolvent Precipitation of Hydrophobic Pharmaceutical Nanoparticles in a Microchannel Reactor, Ind. Eng. Chem. Res., 46 (2007) 8229-8235.

[13] H.-X. Zhang, J.-X. Wang, L. Shao, J.-F. Chen, Microfluidic Fabrication of Monodispersed Pharmaceutical Colloidal Spheres of Atorvastatin Calcium with Tunable Sizes, Ind. Eng. Chem. Res., 49 (2010) 4156-4161.

[14] L. Gao, D. Zhang, M. Chen, Drug nanocrystals for the formulation of poorly soluble drugs and its application as a potential drug delivery system, J. Nanopart. Res., 10 (2008) 845-862.

[15] S. Barcikowski, F. Devesa, K. Moldenhauer, Impact and structure of literature on nanoparticle generation by laser ablation in liquids, J. Nanopart. Res., 11 (2009) 1883-1893. [16] J.P. Sylvestre, A.V. Kabashin, E. Sacher, M. Meunier, J.H. Luong, Stabilization and size control of gold nanoparticles during laser ablation in aqueous cyclodextrins, J. Am. Chem. Soc., 126 (2004) 7176-7177.

[17] S. Besner, A.V. Kabashin, M. Meunier, Fragmentation of colloidal nanoparticles by femtosecond laser-induced supercontinuum generation, Appl. Phys. Lett., 89 (2006) 233122. [18] Y. Tamaki, T. Asahi, H. Masuhara, Tailoring nanoparticles of aromatic and dye molecules by excimer laser irradiation, Appl. Surf. Sci., 168 (2000) 85-88.

[19] Y. Tamaki, T. Asahi, H. Masuhara, Nanoparticle Formation of Vanadyl Phthalocyanine by Laser Ablation of Its Crystalline Powder in a Poor Solvent, J. Phys. Chem. A, 106 (2002) 21352139.

[20] T. Asahi, T. Sugiyama, H. Masuhara, Laser Fabrication and Spectroscopy of Organic Nanoparticles, Accounts Chem.Res., 41 (2008) 1790-1798.

[21] B. Li, T. Kawakami, M. Hiramatsu, Enhancement of organic nanoparticle preparation by laser ablation in aqueous solution using surfactants, Appl. Surf. Sci., 210 (2003) 171-176. [22] H.G. Jeon, T. Sugiyama, H. Masuhara, T. Asahi, Study on electrophoretic deposition of size-controlled quinacridone nanoparticles, J. Phys. Chem. C, 111 (2007) 14658-14663.

[23] J. Hobley, T. Nakamori, S. Kajimoto, M. Kasuya, K. Hatanaka, H. Fukumura, S. Nishio, Formation of 3,4,9,10-perylenetetracarboxylicdianhydride nanoparticles with perylene and polyyne byproducts by $355 \mathrm{~nm}$ nanosecond pulsed laser ablation of microcrystal suspensions, J.Photochem. Photobiol. A-Chem., 189 (2007) 105-113.

[24] P. Wagener, S. Barcikowski, Laser fragmentation of organic microparticles into colloidal nanoparticles in a free liquid jet, Appl. Phys. A-Mater. Sci. Process., (2010) doi:10.1007/s00339010-5814-X.

[25] T. Sugiyama, T. Asahi, H. Masuhara, Formation of $10 \mathrm{mn}$-sized oxo(phtalocyaninato)vanadium(IV) particles by femtosecond laser ablation in water, Chem. Lett., 33 (2004) 724-725. 
[26] S. Kenth, J.-P. Sylvestre, K. Fuhrmann, M. Meunier, J.-C. Leroux, Fabrication of Paclitaxel Nanocrystals by Femtosecond Laser Ablation and Fragmentation, J. Pharm. Sci., (2010) DOI $10.1002 /$ jps.

[27] Z.-B. Zhang, Z.-G. Shen, J.-X. Wang, H. Zhao, J.-F. Chen, J. Yun, Nanonization of Megestrol Acetate by Liquid Precipitation, Ind. Eng. Chem. Res., 48 (2009) 8493-8499.

[28] A. Schnoor, S. Petersen, S. Barcikowski, Laserfragmentierung von anorganischen und organischen Mikropartikel-Suspensionen zu Nanopartikel-Kolloiden, Chem. Ing. Tech., 82 (2010) 317-326.

[29] A. Chow, H. Tong, P. Chattopadhyay, B. Shekunov, Particle Engineering for Pulmonary Drug Delivery, Pharm. Res., 24 (2007) 411-437. 
Supporting Material
Click here to download Supplementary Material: SI-Sylvestre-100810.docx

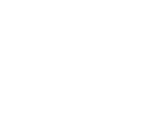

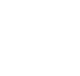

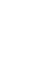

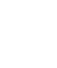

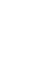

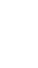

.

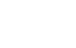

更

更

更

更

更

更

更

更

更

更

更

更

更

更

更

更

更

更

更

更

更

更

更

更

更

更

更 